\title{
Análisis socioeconómico en la provincia de Huelva en las elecciones de 1933.
}

CRISTOBAL GARCIA GARCIA

\section{INTRODUCCION: LAS ELECCIONES DE 1933 EN ESPAÑA}

Las elecciones del otoño de 1933 constituyen un campo privilegiado para la investigación del bistoriador ${ }^{1}$ Esta afirmación realizada en 1976, hace 15 años, se contradice con el abandono por parte de la historiografía sobre estos acontecimientos. Este abandono se manifiesta claramente en el hecho de que no exista ninguna monografía sobre las elecciones de 1933 para el conjunto de España. Sería pretencioso intentar explicar aquí las razones por las que los historiadores han preferido el estudio de los procesos electorales de la primera y última etapa de la II República desconsiderando en gran parte los de 1933. Tal vez resultaran más interesantes aquellos acontecimientos que supusieron el inicio y el final de un sistema político. Tal vez las dificultades para hacer un balance de los resultädos de 1933 fruto de la propia ley electoral, de la diversidad de coaliciones o de los cambios que tras las elecciones se produjeron en los grupos parlamentarios han contribuido a ello. Estas dificultades sin embargo hacen más atractivo el análisis provincial de estas elecciones en la medida en que pueden clarificar el panorama nacional o matizar algunas de las generalidades que sistemáticamente se reproducen.

Dentro de los estudios generales sobre la II República todos los autores coinciden en subrayar dos aspectos: las elecciones de otoño de 1933 van a suponer un punto de inflexión en el ritmo de la vida política española y se va a agudizar, como resultado del propio proceso electoral y de los acuerdos posteriores a las elecciones, la bizcírización en el parlamento y en la sociedad entre derechas e izquierdas.

A esa bipolarización en el panorama político y a esa inflexión en la historia de la República contribuyó también la propia ley electoral. Es

(1) MONTERO, J.R. (1988): -Las derechas en el sistema de partidos del segundo bienio républicano: algunos datos introductorios. en La' II República Española. Bienio rectificador y Frente Popular, 1934-1936. pp: 3-35. Siglo' XXI. Madrid. 
interesante resaltar aquí alguno de los juicios de José Ramón Montero ${ }^{2}$ sobre este aspecto para contrastarlos posteriormente con el caso de Huelva:

1. La ley electoral forzaba coaliciones de partidos superando importantes diferencias ideológicas entre ellos.

2. Esta necesidad de coaligarse para aspirar a la mayoría hizo que los partidos menores obtuvieran un número desproporcionado de candidatos debido a la utilidad marginal de esos votos adicionales.

3. La ley facilitaba una enormemente desproporcionada atribución de escaños a las mayorías (en el caso de Huelva un $71 \%$ ), lo que conllevaba una sobrerrepresentación de la mayoría al traducir los votos en escaños.

Estos aspectos de la propia ley electoral, como ya se ha afirmado, contribuyeron a producir un vuelco en el panorama político en tan solo dos años. La aglutinación de la derecha olvidando importantes diferencias estratégicas e ideológicas impulsó una apasionada y muy ideologizada campaña electoral que en buena medida condicionó los resultados. Ese apasionamiento se manifestó por una parte en el discurso ideológico antimarxista por parte de la derecha que quería hacer aparecer a los socialistas como los únicos artífices de la obra revolucionaria del primer bienio, y por otra parte en el llamamiento a la movilización del voto obrero por parte de los socialistas para frenar la avalancha reaccionaria. Todas estas características se agudizaron entre la primera y la segunda vuelta en aquellas circunscripciones en que ningún candidato alcanzó en primera instancia el quorum del $40 \%$. Hasta aquí los juicios de José $\mathbf{R}$. Montero. Tuñon de Lara afirma en la obra citada que la segunda vuelta consumó las alianzas y triunfos de derechistas y radicales con las únicas excepciones de Madrid Capital y Málaga.

Dentro de la tónica, necesariamente general por breve, que se ha presentado sobre el carácter y el sentido de las elecciones de 1933 en España, se debe concluir este Capítulo adelantando aquellas especificidades que se producen en este momento en la provincia de Huelva frente a la generalidad de España y que por sí mismas justifican este trabajo:

1. Si la ley electoral forzaba coaliciones de partidos superando importantes diferencias ideológicas y si esta afirmación se puede aplicar sobre todo a la derecha en el intervalo de tiempo entre la primera y la segunda vuelta, en Huelva es precisamente en ese espacio de tiempo cuando se rompe la coalición de derechas, si bien recreando una nueva.

(2) Básicamente, para este Capítulo se ha partido del trabajo de ALVAREZ REY L. y RUIZ SANCHEZ, J.L. (1990): Huelva durante la segunda República: Partidos, Elecciones y Comportamiento político (1931-1936). en Huelva en su Historia 3, pp.603-670. Publicaciones Colegio L.iversitario de La Rábida. En este capítulo, los autores recogen los prolegómenos de las elecciones de noviembre de 1933. 
2. Si en gran parte de España son los grupos de la CEDA los que hegemonizan la presencia de las derechas y fuerzan la coalición', fundamentalmente con los radicales, en Huelva esa coalición que surge entre la primera y segunda vuelta va a ser hegemonizada por los conservadores de Maura llevando como socios a los radicales y excluyendo a A.P.

3. Si la ley electoral atribuía un desproporcionado reparto de escaños a las mayorías y este hecho benefició fundamentalmente a las derechas, en Huelva ese beneficio recayó en los socialistas a pesar de que el electorado fuera mayoritariamente de derechas.

Estos tres aspectos que hasta ahora sólo se ha apuntado, van a constituir el centro de atención del siguiente capítulo.

\section{LAS ELECCIONES EN HUELVA: NEGOCIACIONES, CANDIDATURAS Y RESULTADOS ELECTORALES}

\subsection{De la primera a la segunda vuelta}

La primera vuelta de las elecciones generales, celebrada el día 19 de noviembre de 1933, sirvió para decantar en la provinciá de Huelva a aquellos candidatos y a los grupos políticos que tuvieran alguna opción ante el electorado onubense.

Del amplio abanico de grupos políticos que se presentaron en 'esta primera instancia sólo llegarían a la segunda vuelta el 3 de diciembre, un partido político, el $P S O E$; una Coalición Republicana de Centro-Derecha, integrada por tres conservadores (mauristas) y dos radicales (de Lerroux); ambos con candidatura completa (cinco nombres); y junto a ellos, un grupo político, Acción Popular (de Gil Robles), que habiendo formado parte de la Coalición de Derechas con los mauristas en la primera vuelta, presentaría ahora un único candidato.

En estas tres opciones se reagrupaban once de los quince candidatos que el 19 de noviembre superaron el $8 \%$ de los votos sin haberse proclamado ninguno al no alcanzar el $40 \%$ de los votos válidos. ${ }^{3}$

(3) En la segunda vuelta, Sánchez-Dalp perdería 15.198 votos, el el 34,8\% de los obtenidos en la primera. En algunos distritos, no obstante, aumentaría el númeró de votos. Es significativo que en el Partido Judicial de Aracena sacara el 3 de diciembre 11.116 votos, siendo el candidato de derechas más votado. Efectivamente Sánchez-Dalp tenía una gran fuerza electoral en la Sierra. Conviene recordar que D. Manuel Sánchez-Dalp es el heredero en Aracena de un cacicato que viene de la Restauración. TUSELL GOMEZ, J.(1976): Oligarquía y Caciquismo en Andalucia (1980-1923).p. 243 Planeta. Barcelona, define a este cacicato como estable, conservadory muy poco competitivo. De las múltiples referencias que tiene de esta fa ilia, entresacamos de la p.291 el hecho de que ostentaban los títulos de Marques de $\mathrm{A}$ acena y Conde de Torres de Sánchez-Dalp, concedidos por Alfonso' XIII. Se trata pues de una familia de raigambre caciquil, versada en la política desde el siglo pasado. 
El resto, radicales socialistas, federales y comunistas no superaron el listón del $8 \%$ y consecuentemente no pasaron a la segunda vuelta.

Los resultados de la primera vuelta y las negociaciones que ocuparon el tiempo hasta la presentación de las candidaturas para la segunda, tuvieron una importancia decisiva de cara a los resultados finales del 3 de diciembre rompiendo todas las previsiones que apuntaban a una bipolarización entre socialistas y otra candidatura *antimarxista* de derechas.

Al final, la desunión de las derechas impidió en primer lugar que el candidato de Acción Popular (Manuel Sánchez Dalp) saliera elegido y en segundo lugar permitió que la candidatura socialista superara en diputados a la Coalición de Centro-Derecha por cuatro a tres.

Estos dos efectos eran fácilmente previsibles a la luz de los primeros resultados del 19 de noviembre y del ambiente general que se respiraba en la derecha española, que solamente unida acabaría desalojando a los socialistas del gobierno de la República.

\subsection{La ruptura de la COALICION DE DERECHAS y la formación de la COAIICION REPUBLICANA DE CENTRO-DERECHA}

¿Qué razones entonces llevaron a esa ruptura entre el Partido Republicano Conservador y Acción Popular en la provincia de Huelva?

En primer lugar para responder a esta pregunta hay que remontarse a los orígenes de la formación de la Coalición de Derechas. El 4 de octubre de 1933, A.P. se dirige a la opinión pública a través de una nota en el Diario de Huelva en la que da cuenta del acuerdo de su Comité Provincial de designar dos candidatos para las minorías: D. Mariano Pérez de Ayala, Delegado Provincial de A.P. en Huelva y D. Manuel Sánchez-Dalp y Marañón, de A.P. serrana.

Posteriormente, el 31 de octubre, los conservadores confeccionaron la Coalición de Derechas con tres miembros del P.R.C. (Dionisio Cano, Pérez de Guzmán y Jiménez Molina) y un independiente de derechas (Manuel Fernández Balbuena). En la candidatura se deja un puesto para A.P. La coalición veía con simpatía que ese puesto lo ocupara el Sr. Sánchez Dalp por la fuerza electoral que tenía en la Sierra.

A.P. aceptó ese puesto, pero quedaría con el resquemor de no haber podido incluir a sus dos candidatos en la Coalición. Las susceptibilidades que provocó la renuncia al segundo candidato de A.P. entre sus afiliados y simpatizantes, debieron ser muchas. Prueba de ello es que la Dirección se vio obligada a hacer repetidos llamamientos a la opinión publica. Reproducimos el primero de ellos publicado en el Diario de Huelva el 5 de noviembre:

.Teniendo conocimiento este Comité Provincial de ciertos rumores propalados que parecen significar desagrado por parte de elementos 
afiliados a nuestra organización y en contra de la Candidatura de la Coalición de Derechas hace constar que Acción Popular de Huelva respeta en absoluto el pacto electoral existente esperando de todos sus afiliados la más eficaz colaboración.*

El 16 de noviembre A.P. tendrá que salir al paso desautorizando rumores difundidos por elementos ajenos a esta organización que atacan a Maura y aconsejan se vote sólo a la representación de A.P

Todo hace pensar que existian fisuras en la Coalición de Derechas y una causa de esas fisuras era el descontento de A.P.por la exclusión en la candidatura.de D. Mariano Pérez de Ayala.

Para seguir avanzando en el por qué de la ruptura es preciso pararse en un somero análisis de los resultados del 19 de noviembre. SánchezDalp, en la Coalición de Derecbas consiguió 43.624 votos. Se puede afirmar que los resultados de A.P. estuvieron por encima de la solitaria presencia de Sánchez-Dalp junto a los conservadores. Y no sólo esto, ese número de votos se sitúa a 11.253 por encima de los obtenidos por Fernando Rey Mora y a 11.491 por encima de los obtenidos por José Terrero, ambos candidatos radicales que más votos obtuvieron en esta primera vuelta y que posteriormente formarían parte de la Coalición para la segunda vuelta, que pasaría a llamarse Coalición Republicana de Centro-Derecha. ${ }^{4}$

Con este bagaje de 43.624 votos en la primera vuelta, A.P. podría permitirse jugar fuerte en la Coalición para la segunda intentando mermar la hegemonía que tuvieron los conservadores en las primeras negociaciones, pensando que en cualquier caso mantendrían esa fuerza electoral el 3 de diciembre.

Sobre la versión oficial de la ruptura, existen cuatro comunicados de prensa que entre los días 9 de noviembre y 2 de diciembre. Por razones de espacio reproducimos solamente el primer comunicado:

Acción Popular (29 de noviembre) ${ }^{5}$

-Acción Popular que con todo entusiasmo y lealtad, defendió en las elecciones del día 19 la candidatura íntegra de la Coalición de Derechas, se ve ahora, desplazada de la que a base de algunos elementos de aquella se ha formado para la próxima contienda electoral... Ya lo saben

(4) El texto íntegro de esta primera nota de A.P. está recogido en el trabajo citado de Alvarez Rey y Ruiz Sánchez. Ellos lo toman del Diarrio La Unión que lo publicó el día 30 de noviembre.Los otros comunicados redactados en tono de réplica y contrarréplica no contribuyen a aclarar muchas cosas.

(5) Evidentemente no se puede pretender dar por cerrada toda explicación a esa ruptura. Esta aproximación al tema se ha limitado a la lógica de los datos y a las versiones oficiales. Pero esto es compatible con otras explicaciones como la señalada por Alvarez Rey y Ruiz Sánchez sobre el arbitraje de Burgos y Mazo a quien adjudican la autoría del rechazo a A.P. 
pues, nuestros afiliados de A.P., lanzada de la candidatura de la Coalición afronta con sus solas fuerzas y pide a todos el máximo esfuerzo para lograr el triunfo de su candidato."

Una nota del Secretario de A. P. de Valverde del 1 de diciembre va a ser mucho más explícita al manifestar que los conservadores quieren -imponer una candidatura y que en su origen en la Coalición de Derechas se deberían haber incluido dos miembros de A.P. en detrimento de un conservador. Acabará pidiendo el voto ssólo* para Sánchez-Dalp.

Con todos estos datos y volviendo a la pregunta inicial sobre la ruptura de las derechas en Huelva, podemos concluir:

1. Conservadores $y$ A. $P$. defienden públicamente y con entusiasmo la necesidad de ampliar la Coalición, que al final se rompe.

2. Existieron problemas de entendimiento entre $P R C$ y $A P$ en las negociaciones para la segunda vuelta y esos problemas dieron al traste con el posible acuerdo de los tres partidos.

3. A.P. nunca aceptó en su fuero interno la hegemonía de los conservadores en la Coalición. Los buenos resultados de Sánchez-Dalp en la primera vuelta les impulsó a conseguir un mayor protagonismo en la negociación encaminada a ampliar la Coalición. A.P. acabaría sintiéndose desplazada de la Coalición, extremo que niega el P.R.C.

4. El P.R.R. (Radicales), aparecen al margen de los problemas de la negociación.

5. A.P. aspirará en la segunda vuelta, reforzada por los resultados del 19 de noviembre, a conseguir el Acta de Diputado para SánchezDalp, restando votos a la Coalición de Centro-Derecha.

Junto a estas conclusiones quedan abiertas las preguntas fundamentales: ¿Cuál fue y de qué índole la razón verdadera de la ruptura? ¿Por qué esa o esas razones no se suscitaron antes de la primera vuelta? ¿Tuvo que ver o no con esa ruptura el intento de abrir la Coalición al Centro? ${ }^{6}$

El 29 de noviembre de 1933 se hace pública la Coalición Republicana de Centro Derecha.

\subsection{Los resultados electorales. Un primer análisis}

El día 7 de diciembre, la JUNTA PROVINCIAL DEL CENSO hace públicos los resultados de las votaciones a efectos de la proclamación de los Diputados a Cortes:

(6) FUENTES: ARCHIVO DE LA DIPUTACION PROVINCLAL DE HUELVA: Junta Provinciai del Censo Electoral. Huelva. Elecciones para Diputados a Cortes. 1933. EXPEDIENTE GENERAL. (LEGAJO 85). Diario LA PROVINCIA (8 diciembre 1933). DLARIO DE HUEIVA (7 diciembre 1933). El orden de los candidatos de más a menos votados es nuestro. 
Candidatura Radical-Conservadora

Fernando Rey Mora $63.862 \quad$ (PRR)

Francisco Pérez de Guzmán 62.620 (PRC)

Dionisio Cano López $60.914 \quad$ (PRC)

José M. Jiménez Molina 59.529 (PRC)

José Terrero Sánchez 57.159 (PRR)

Partido Socialista

Crescenciano Bilbao $\quad 60.137$

Juan Tirado Figueroa $\quad 59.937$

Amós Sabrás Gurrea $\quad 59.921$

Ramón González Peña $\quad 59.788$

Antonio Ramos Oliveira 59.610

Acción Popular

Manuel Sánchez Dalp 28.426

Se proclamaron Diputados los Sres. Rey Mora, Pérez de Guzmán, Cano López de la coalición radical-conservadora, y los Sres. Bilbao, Sabräs, Tirado y Peña del partido socialista. ${ }^{\text {? }}$

Se impone una primera lectura de estos resultados a la vista del análisis anterior sobre la división de la derecha.

La diferencia de votos entre el último diputado electo, el socialista González Peña y el primer candidato de derechas no electo, el conservador Jiménez Molina, es de tan sólo 259 votos. Por último, la diferencia de votos entre Amós Sabrás y José Terrero Sánchez, candidato radical no electo, es de 2.762 votos. Por último, la diferencia de votos entre Amós Sabrás y Jiménez Molina es de 392 votos.

Podemos afirmar pues, que esos 28.426 votos de Sánchez-Dalp evitaron que los candidatos de derechas Jiménez Molina y José Terrero obtuvieran el acta de diputado.

Un análisis pormenorizado por distritos en la segunda vuelta nos lleva a la conclusión de que en aquellas mesas donde Sánchez-Dalp mantiene a aumenta los votos es siempre a costa de la Coalición.

(7) Un problema metodológico es cómo contabilizar por partidos los votos en unas elecciones abiertas. Diferentes autores proponen diferentes soluciones: la media, la moda, aplicar al partido el resultado del candidato más votado. La utilización de la media es denostada por algunos autores porque se trataría de una mera abstracción, no existen $60.816,8$ votos. Nos parece que cualquier medida es una mera abstracción ya que no existen, y así lo quiso el legislador, votos a partidos sino votos a candidatos. Por otra parte en Estadistica necesariamente trabajamos con medidas que son una abstracción de la realidad. En este caso lo que la media explica es que el electorado de la circunscripción de Huelva era mayoritariamente de derechas. $Y$ cualquier otra medida corroboraría esta afirmación. 
Pero aún hay más, la media de votos de los socialistas es de 59.879 y la de la coalición de $60.816^{8}$.

Si comparamos estas medias con el número de diputados electos en cada candidatura debemos concluir que los socialistas ganan en diputados porque su electorado se comporta más homogéneamente que el electorado de derechas y que ello fue consecuencia de la tercera candidatura en discordia, la de A.P.

Pero además hay índices que nos permiten corroborar esto. La desviación típica en el caso de la media de votos de los candidatos socialistas es de 174,68 y en el caso de la coalición es de $2.348,2$. Esta diferencia indica una mayor dispersión del voto en el segundo caso con respecto al primero. ${ }^{9}$

Concluyendo, los socialistas obtienen cuatro actas de diputados frente a las tres que obtiene la candidatura de centro-derecha como consecuencia de la división de la derecha.

Este Capítulo que se ha centrado en el análisis de la división de las derechas, resulta imprescindible a la hora de interpretar los resultados electorales de 1933 en la provincia de Huelva. A esta necesidad se ha sacrificado haber abordado otros temas de interés como la formación de todas las candidaturas, la implantación de los partidos políticos en Huelva o el estudio de los acontecimientos que precedieron a estas elecciones.

\section{OBJETIVOS. FUENTES Y METODOLOGIA}

\subsection{Objetivos}

Este trabajo se engloba en un proyecto de investigación más amplio, que pretende un análisis político de las elecciones de 1933 en la provincia de Huelva. Algunas conclusiones se han adelantado ya en los dos primeros capítulos pero considerando siempre a la provincia de Huelva

(8) Para hallar la desviación típica hemos procedido así: se calcula la diferencia de cada resultado con la media. Estas diferencias se elevan al cuadrado, se suman los cuadrados y se divide por el número de candidatos y extraemos la raíz cuadrada del resultado. Este cálculo se puede completar con el Coeficiente de Variación (C.V.) que no es sino el número de veces que supone la desviación típica respecto a la media. Se calcula dividiendo la desviación típica por la media y multiplicando el resultado por cien. El C.V. en el caso de la candidatura socialista es de $0,29 \%$ y en la coalición es de $3,86 \%$. En ambos casos se trata de valores poco dispersos con relación a la media. Pero esa homogeneidad es mayor en los candidatos socialistas y es cualitativamente mayor porque proporcionó una mayoría en diputados a una minoría en votos.

(9) Para la aplicación de estas Técnicas nos hemos basado fundamentalmente en los siguientes textos: SIERRA BRAVO, R. (1989) 6 edic. Técnicas de Investigación Social. Teoria y Ejercicios. Paraninfo. Madrid y PUIDDO SAN ROMAN, A. (1976) 4 edic. Estadística y Técnicas de Investigación Social. Pirámide. Madrid. 
en su conjunto. Un estudio más detenido de los resultados por comarcas nos permitirá un acercamiento a la realidad onubense en su diversidad en las elecciones de 1933.

Lo primero que sorprende cuando se empieza a considerar la heterogeneidad del comportamiento electoral en el conjunto de la provincia es el antagonismo entre unas comarcas y otras. Así mientras la Capital tiene un comportamiento más semejante al conjunto de la provincia; la Costa y el Condado se inclinan por el Centro-Derecha y las zonas mineras y en gran parte la Sierra votan socialista.

¿Qué sectores sociales hacen que el voto se incline por una u otra opción? ¿Por qué los socialistas en determinadas comarcas no se desgastan ante el electorado entre 1931 y 1933? ¿Qué acontecimientos y de qué índole (política, social, económica) pudieron influir en ello? A estas preguntas y otras que pudieran formularse a lo largo del proceso de investigación, se pretende ir contestando.

Ya se ha señalado el objetivo general del trabajo y el marco en que se engloba, se trata a continuación de fijar una serie de objetivos específicos:

1. Llegar a un conocimiento de la implantación del voto de los candidatos políticos por comarcas.

2. Naturalmente no podemos cruzar variables sociodemográficas con el voto, dado el carácter secreto de éste, pero las variables sociodemográficas si pueden cruzarse entre sí en análisis bivariables que nos permita una caracterización más a fondo de cada comarca. De este modo, a priori, tendríamos una buena descripción por comarcas.

3. Una vez realizada esta descripción, comarca a comarca se podría establecer una ordenación general de las comarcas en base a las opciones políticas. Este objetivo se concretaría en una comparación cualitativa de las diferencias observadas comarcalmente (y por tanto de las opciones políticas).

4. A partir de aquí se plantea la siguiente hipótesis de trabajo: ¿Qué relación existe entre una relativa homogeneidad de las variables sociodemográficas con las preferencias políticas? O, si distintas (opuestas) preferencias políticas muestran heterogeneidad en relación a esas variables. Asimismo constatar si en aquellas comarcas donde el voto se equilibra, también lo hacen las variables sociodemográficas.

\subsection{Fuentes y metodologia}

\subsubsection{El censo electoral}

Del Archivo de la Diputación Provincial de Huelva se ha utilizado el Censo Electoral ordenado por Decreto de 26 de Enero de 1932.

Al final de la relación correspondiente a cada sección electoral aparece una lista adicional de los residentes en la demarcación de la 
expresada seċción que adquirirán el derecho de sufragio antes del $1^{\circ}$ de Noviembre de 1933. La utilización por tanto de esta fuente documental obliga a alguna matización que no resta rigor al estudio.

Convocándose las elecciones el 9 de Octubre y celebrándose en primera vuelta el 19 de Noviembre y en segunda vuelta el 3 de Diciembre habría que excluir a aquellos electores fallecidos desde la confección del censo hasta la celebración de las elecciones y habría que incorporar a aquellos que adquirieran su derecho al sufragio entre el 1 y el 19 de Noviembre.

Estas matizaciones no hacen estadísticamente significativa la diferencia entre el censo con el que trabajamos y los ciudadanos con derecho a voto en las elecciones de 1933. Pero menos significativa es aún esa diferencia a efectos de obtener un perfil socioeconómico de la población de la provincia de Huelva en el momento de realizarse esas elecciones.

El Censo Electoral se nos presenta de la siguiente manera: dividido en MUNTCIPIOS, por orden alfabético, cada Municipio dividido en DISTRITOS, cada distrito en SECCIONES, y en cada Sección la relación alfabetizada de los ELECTORES seguida de la lista adicional donde se relacionan también alfabéticamente los jóvenes que cumplirán los 23 años antes del 1 de noviembre.

De cada elector se nos ofrecen los siguientes datos: NOMBRE, DOMICILIO, SEXO, EDAD, PROFESION, SABE LEER Y ESCRIBIR/NO SABE.

\subsubsection{Los resultados electorales}

Del Archivo de la Diputación Provincial de Huelva, se ha trabajado con el siguiente documento: Junta Provincial del Censo Electoral. Huelva. Elecciones para Diputados a Cortes. 1933. EXPEDIENTE GENERAL. (Legajo 85). Este legajo está muy incompleto. No obstante, encontramos una relación de votos de los candidatos (tanto en la primera como en la segunda vuelta) que por su presentación responde a lo que fue el recuento definitivo por secciones electorales y candidatos. Dado que los resultados coinciden básicamente con los oficiales publicados entonces por la Prensa, hemos considerado su utilización en el presente trabajo.

\subsubsection{El tratamiento de las fuentes}

Siendo la población total del Censo que nos ocupa, para toda la provincia de Huelva, de 200.610 electores, es obvia la utilización de Técnicas de Investigación Social, que nos permitan seleccionar datos y aplicar fórmulas que hagan posible llegar a resultados. ${ }^{10}$

(10) La utilización de esta Tabla nos evita el tener que aplicar la fórmula del muestreo para cada caso. Además, el resultado de la aplicación de la fórmula es siempre algo menor que el que nos da la Tabla, por lo que la utilización de esta no resta rigor. Los márgenes 
La primera cuestión que se plantea es como subdividir el conjunto de la población en base a los objetivos propuestos. Se ha optado por un criterio de comarcalización atendiendo a las características económicas, fundamentalmente la producción, criterio que como se verá más adelante se ha seguido también en la codificación de las categorías profesionales, introducciendo alguna corrección como separar el Andévalo en Oriental y Occidental a efectos de distinguir entre aquellos núcleos mineros importantes con más tradición de lucha y organización obrera de otros más pequeños, por la importancia que ello pudiera tener en el comportamiento electoral.

De esta manera nos encontramos con 7 sectores de población, agrupados en las siguientes comarcas:

$\begin{array}{lr}\text { COMARCA } & \text { POBLACION } \\ \text { Huelva Capital } & 22.163 \\ \text { Costa } & 21.274 \\ \text { Condado } & 37.548 \\ \text { Campiña Baja } & 19.158 \\ \text { Andévalo Oriental } & 40.221 \\ \text { Andévalo Occidental } & 17.622 \\ \text { Sierra } & 42.624 \\ \text { TOTAL } & 200.610\end{array}$

Cada una de estas comarcas, a efectos estadísticos, la consideramos como la población sobre la que trabajar $(N)$.

Para la selección de la muestra ( $n$ ) en cada una de estas comarcas, primer paso para el tratamiento estadístico, acudimos a la .Tabla para la determinación de una muestra sacada de una población finita, para un margen de error del $\pm 4 \%$ y con un margen de confianza del 95\% (SIERRA' BRAVO, R. 198:-234). ${ }^{11}$ Obteniendo en cada caso las siguientes muestras:

de error y confianza que se han seleccionado son los que se utilizan normalmente para estudios electorales. El margen de error nos define la divergencia entre los valores medios estadísticos de la muestra en relación al universo o población. Este error determina un intervalo de confianza; y el margen de confianza a su vez el grado de probabilidad de que la media del universo esté en ese intervalo de confianza.

(11) Por ejemplo, en el caso de Huelva Capital la fracción de muestreo es de 0,027 (610/ 22.163). Esta fracción multiplicada por el número de electores de cada sección nós dará el número de individuos a tomar por cada una de las secciones. Así, si en la Mesa electoral 1 del Distrito Municipal 1 aparecen 619 electores; procederemos: 619 x 0,027 = 17. Serán 17 los electores a elegir en esa mesa para el muestreo. Es decir, repartimos la muestra de forma proporcional a cada municipio y dentro de él a cada sección electoral. Se procede así porque en el caso que nos ocupa, los universos no son homogéneos, dado que cada sección electoral determina agrupamientos de personas que viven en un entorno común (pueblo, barrio dentro de un pueblo o agrupación de calles) que puede determinar características socioeconómicas homogéneas en ese vecindario. Se evita de esta manera el que unos estratos estuvieran más o menos representados que lo proporcionalmente debido. 


\section{COMARCA}

Huelva Capital

Costa

Condado

Campiña Baja

Andévalo Oriental

Andévalo Occidental

Sierra
POBLACION $(\mathrm{N})$

22.163

21.274

37.548

19.158

40.221

17.622

42.642
MUESTRA (n)

para un margen de error $4 \%$

610

610

617

606

617

606

617

El siguiente paso consistirá en obtener, siempre en cada uno de los siete pasos, la fracción de muestreo, esto es, el resultado de dividir la muestra (n) entre la población o universo $(\mathrm{N}) .^{12}$

Una vez que hemos determinado el número de individuos en cada sección electoral, se eligen éstos por azar simple. Puesto que los individuos aparecen con un número consecutivo, adjudicamos un número correlativo a los electores que aparecen en la lista adicional. Los electores para la muestra se seleccionan utilizando las Tablas de Fisher y Yates (SIERRA BRAVO, R. 1989-202). ${ }^{13}$

Para la recogida de las muestras se han confeccionado unas fichas que recogiendo todas las variables (comarca, municipio, distrito, sección, varón/hembra, edad, profesión, sabe leer y escribir/no sabe) nos facilite el posterior tratamiento de los datos.

Pero previamente a la informatización de los datos habrá que enfrentarse a otro problema metodológico, la codificación de las profesiones. Cualquier tipificación socioprofesional puede presentar múltiples objeciones. En los estudios históricos de sociología electoral no encontramos una norma que pueda aplicarse con carácter universal. La principal dificultad proviene de la subjetividad con que los individuos se definen profesionalmente. Otro problema lo plantea la confusión entre la profesión, la ocupación, y en algunos casos la condición de inactivos (impedido).

En el caso que nos ocupa como medida de prudencia, se ha preferido abordar la codificación profesional una vez que recogidos los datos del censo, se tuviera una relación completa, antes que partir de una codificación previa que no garantizara si se iba a ajustar a la realidad, a fin de dejar a la interpretación personal los menos casos posibles, siempre inevitables.

(12) FISHER ET YATES: Stadistical Tables. (Hay una edición en castellano publicada por Edit. Aguilar).

(13) En la Costa se han incluido los siguientes municipios: Ayamonte, Cartaya, Isla Cristina $y$ Lepe.Y en el Andévalo Oriental: Berrocal, Calañas, Campofrí, El Cerro, La Granada de Riotinto, Minas de Riotinto, Nerva, Saluochea (hoy El Campillo), Valverde, Villanueva de las Cruces y Zalamea. 
Una opción, utilizada por muchos autores, hubiera sido la de seguir el criterio del grupo social (burguesía, clase media, proletariado). Pero los datos tal y como se presentan en el Censo Electoral no nos lo posibilita. Nunca se sabe por esta sola fuente, si detrás de la denominación de labrador hay un terrateniente o un pequeño campesino más próximo al proletariado. Lo mismo se podría decir de otras denominaciones (industrial, comerciante). El señalar estas dificultades previas a la codificación, sin embargo no puede llevar a la conclusión de que al final no obtengamos una idea muy aproximada de la estructura social como se verá en el capítulo siguiente.

Pero en definitiva y dado que un criterio básico de comarcalización ha sido la producción, parece acertado tipificar en base a los diferentes sectores de producción, cada uno de los cuales, nos aparecerá como más predominante en cada comarca. Con este criterio y atendiendo: a la necesidad de no hacer una codificación excesivamente amplia, que impida una visión global, se ha confeccionado el siguiente cuadro:

$\begin{array}{ll}\text { CATEGORIAS SOCIOPROFESIONALES } & \text { DENOMINACION EN EL CENSO } \\ \text { Agricultor (Propietario) } & \text { Labrador, Agricultor. } \\ \text { Industrial/Del Comercio } & \text { Industrial, Comerciante. } \\ \text { Prof.Liberal/Funcionario/Universitario } & \text { Catedrático, Maestro, Ingeniero. } \\ \text { Obreros } & \text { Obrero, Chofer, Carpintero, Vigilante, Empleado, etc. } \\ \text { Jornaleros del Campo } & \text { Jornalero, Pastor, Bracero, Campo, Carretero, etc. } \\ \text { Mineros/Jornaleros } & \text { Minero, Jornalero. } \\ \text { Marineros } & \text { Marinero, Motorista, Fogonero, Estivador, etc. } \\ \text { Su Casa } & \text { Su Casa. } \\ \text { Sirvienta } & \text { Sirvienta. } \\ \text { Jubilados } & \text { Jubilado, Impedido, Cesante. } \\ \text { Varios } & \text { Otras denominaciones. }\end{array}$

\section{PRIMEROS RESULTADOS}

Al objeto de completar esta comunicación y de anticipar un trabajo más extenso, se ha querido seleccionar dos comarcas, de entre las que dividimos a la provincia de Huelva, con el fin de ensayar una validación del trabajo en un análisis necesariamente muy breve. Estas comarcas son la COSTA y el ANDEVALO ORIENTAL.

La elección de estas dos comarcas se justifica por el opuesto comportamiento electoral que mantienen en 1933:

El PSOE obtiene en el Andévalo Oriental, una media del $75,30 \%$ de los votos válidos mientras que en la Costa descienden al 12,87\%.

La Coalición de Centro-Derecha obtiene en el Andévalo Oriental una media del $12,94 \%$ mientras que en la Costa ascienden al $73,16 \%$. 
Se trata por tanto de resultados antagónicos y en absoluto representativos del conjunto de la provincia. (Gráfico 1)

Una vez justificada la elección de las comarcas en base a los resultados electorales, se ha procedido a analizar en cada una de ellas alguna de las relaciones bivariables que posibilitan los datos del Censo: SexoAlfabetización, Profesión-Alfabetización, Sexo-Profesión. Todo ello como resultado del proceso de tratamiento sobre las variables de 1.227 electores que suman las muestras de ambas comarcas.

1. La relación SEXO-PROFESION: Al menos la clase social que se define como proletariado aparece claramente delimitada por las siguientes profesiones: Jornaleros del Campo, Marineros, Obreros y Mineros. Atendiéndose a esto, el proletariado de la Costa representa el $88 \%$ y en el Andévalo Oriental el $86,72 \%$. No podemos por tanto afirmar que la Estructura Social determine la orientación del voto si consideramos los resultados electorales en una y otra comarca, pero si que la profesión de minero determina una orientación del voto hacia la izquierda.

La profesión de minero además en el caso del Andévalo Oriental tiene un peso más específico en el conjunto del proletariado, representando el $41,61 \%$, frente al $31,12 \%$ de obreros y el $13,99 \%$ de jornaleros del campo.

En el caso de la Costa, las tres profesiones que definirían el proletariado se reparten más regularmente, un $31,83 \%$ de jornaleros del campo, un $30,87 \%$ de marineros y un $25,40 \%$ de obreros.

En el caso de las mujeres, no aparecen diferencias significativas, predominando notablemente en ambas comarcas la variable su casa (un $89,26 \%$ en la Costa y un $94,26 \%$ en el Andévalo Oriental.

2. Las relaciones SEXO-ALFABETTZACION-PROFESION: En este caso se encuentran acusadas diferencias en el nivel de alfabetización entre una comarca y otra. Si se atiende a los totales, en el Andévalo Oriental saben leer y escribir el $60,45 \%$ de los electores frente al $44,75 \%$ en la Costa. Estas diferencias se hacen más significativas si atendemos a la relación SEXO-ALFABETIZACION, así en el Andévalo Oriental saben leer y escribir el $75,52 \%$ de los varones frente al $48,55 \%$ en la Costa que desciende al 33,33\% en el caso de los marineros. (Gráficos 2, 3, 4 Y 5).

Un mayor nivel de instrucción en los mineros junto con el ya apuntado peso específico en el conjunto del proletariado posibilitaba una mayor conciencia social en el Andévalo Oriental que al margen de otras causas quizás más determinantes (la tradición de organización y lucha en la Cuenca Minera) influía sin duda en esa orientación a la izquierda del voto.

Evidentemente no se han agotado todas las posibilidades de análisis demográfico social y económico en relación con las preferencias políticas, pero puede ser suficiente para validar una metodología de investigación. 


\section{APENDICE.DE GRAFICOS}

Gráfico 1: Elecciones Generales 1933. Resultados Costa y Andévalo Oriental

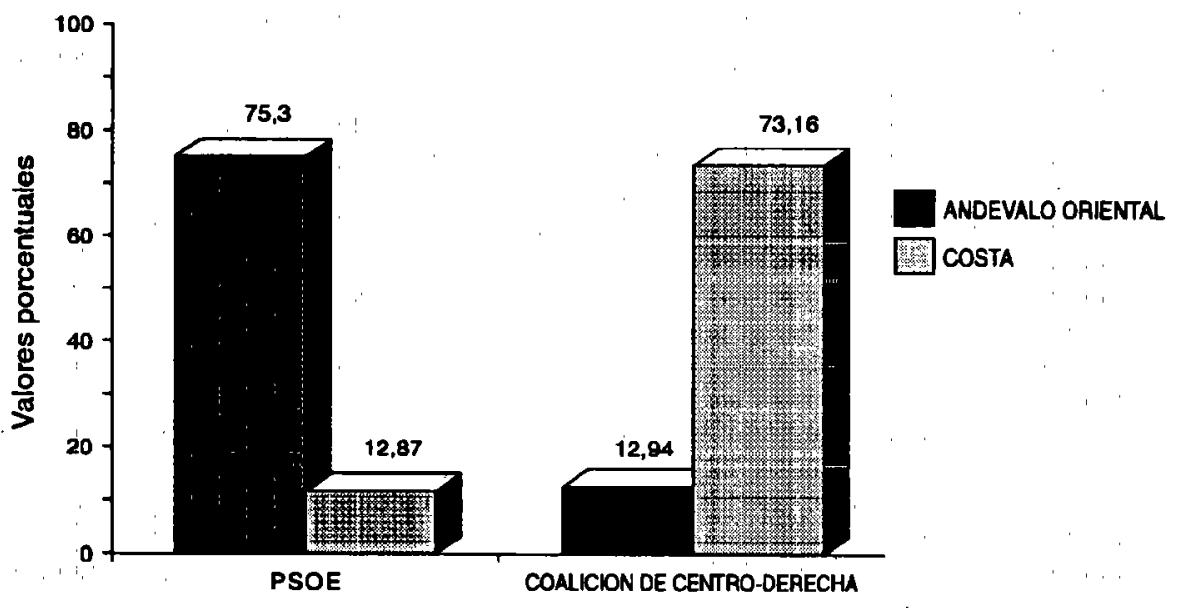

Fuente: Archivo de la Diputación de Huelva. Elaboración propia.

Gráfico 2: Alfabetización electores, Andévalo Oriental

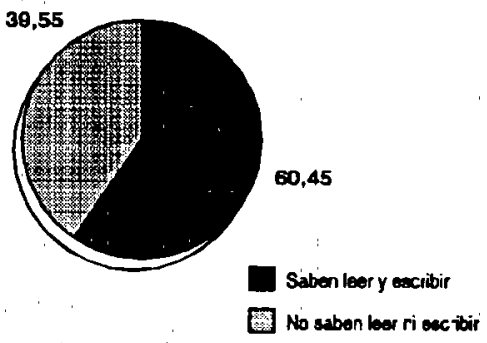

Gráfico 2: Alfabetización mineros, Censo electoral 1933

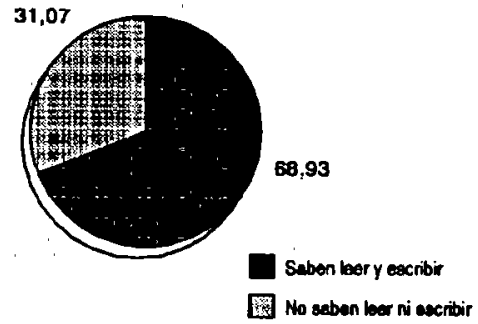

Gráfico 3: Alfabetización electores, Costa

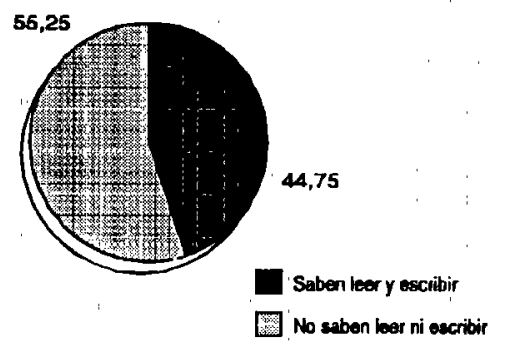

Gráfico 3: Alfabetización marineros, Censo electoral 1933

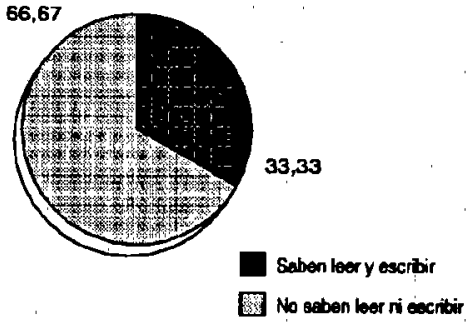

Fuente: Censo Electoral 1933. ADH. Elaboración propia. 


\section{BIBLIOGRAFIA}

\section{BIBLIOGRAFIA GENERAL}

ALVAREZ REY, L. y RUIZ SANCHEZ, J. L. (1990): ·Huelva durante la Segunda República: Partidos, Elecciones y Comportamiento político (1931-1936). Pp. 603-670, en Huelva en su Historia, 3. Colegio Universitario de La Rábida. Huelva.

AVILES FARRE, J. (1988): -Los partidos republicanos de izquierda, 1933-1936. En La II Repüblica Española. Bienio Rectificador y Frente Popular, 1934-36. IV Coloquio de Segovia sobre Historia Contemporánea de España. Siglo XXI. Madrid.

BECARAUD, J. (1967, 1a. edic. 1962): La II Repüblica Española. 1931-1936. Ensayo de interpretación. Taurus. Madrid.

GIL PECHARROMAN (1989): La Segunda República. Historia 16. Madrid.

LINZ J. J. (1976): El sistema de partidos en España. Madrid.

MALEFAKIS, E. (1973): -Los partidos de la izquierda y la Segunda República. En Estudios sobre la Repuiblica y la Guerra Civil Española. Barcelona.

MATEOS RODRIGUEZ, M. A. (1991): .Fuentes y Metodología para el estudio electoral de la Segunda República española. En Revista $A Y E R, 3$, pp. 139-164.

MONTERO, J. R. (1988): -Las derechas en el sistema de partidos del segundo bienio republicano: algunos datos introductorios. Pp 3-35 en La II República Española . Bienio Rectificador y Frente Popular, 1934-36. IV Coloquio de Segovia sobre Historia Contemporánea de España. Siglo XXI.

PULDO SAN ROMAN, A. (1976. 4a.): Estadística y Técnicas de Investigación social. Pirámide. Madrid.

RAMIREZ JIMENEZ, M. (Coord.) (1975): -La agrupación de intereses en la II República: Partidos y Grupos. Pp. 27-49. En Estudios sobre la II Repüblica Española. Tecnos. Colección de Ciencias Sociales. Madrid.

ROBINSON, $R$. (1973): .La República y los partidos de derecha. En Estudios sobre la Repuiblica y la Guerra Civil. Barcelona.

RUIZ MANJON-CABEZAS, O. (1979): •Autoridades locales y partidos políticos en Andalucía durante la Segunda República. En Primer Congreso de Historia de Andalucía. Córdoba. Pp. 423-436.

SIERRA BRAVO, S. (1989 6a.): Técnicas de Investigación Social. Teoría y Ejercicios. Paraninfo. Madrid.

TAMAMES, $R$ (1977.5a. edic.): La República. La era de Franco. Alfaguara. Madrid.

TUÑON DE LARA, M (1976. 3 ed.): La II Repüblica, Vol II. Siglo XXI. Madrid.

TUSELL, J. (1976): Oligarquía y caciquismo en Andalucía (1890-1923). Planeta. Barcelona. VARELA, Santiago (1978): Partidos y Parlamento en la Segunda República. Ariel. Madrid. BIBLIOGRAFIA SOBRE ESTUDIOS LOCALES DE SOCIOLOGIA ELECTORAL

BERMEJO MARTIN, F. (1984): La II Repüblica en Logroño: Elecciones y Contexto Político.

Instituto de Estudios Riojanos. Logroño.

CARO CANCELA, D. (1987): La Segunda Repüblica en Cádiz. Elecciones y Partidos Poláticos. Diputación Provincial de Cádiz.

GERMANZUBERO, L. (1984): Aragón en la II República. Estructura económica y comportamiento político. Institución Fernando el Católico. Zaragoza.

GONZALEZ CASANOVA, J. A. (1969): Elecciones en Barcelona (1931-1936). Tecnos. Madrid. MAJUELO GIL, E. (1986): La II República en Navarra. Conflictividad agraria en la Ribera Tudelana (1931-1933). Pamiela Argitaletxea.

MARCOS DEL OLMO, M. (1986): Las elecciones del Frente Popular en Valladolid. Diputación Provincial de Valladolid.

MARTIN VASALLO, J. R. (1982): Las Elecciones a Cortes en la Ciudad de Salamanca (1931-1936). Un estudio de sociología electoral. Servicio de Publicaciones del Ayuntamiento de Salamanca. 
OLIVER ARAUJO, Joan (1983): La II República en Baleares: Elecciones y Partidos Políticos (Ensayo de Sociologia Electoral Balear). Institut D'Estudis Baleàrics. Paima de Mallorca. PABLO CONTRERAS, Santiago de (1989): La II República en Alava. Elecciones, partidos y vida política. Servicio Editorial. Universidad del País Vasco.

SANCHEZ SANCHEZ, J y MATEOS RODRIGUEZ, M. A. (1977): Elecciones y Partidos en Albacete durante la II Repüblica 1931-1936. Análisis demográfico. Actividad económica. Sociología Electoral y comportamiento político. Julián Gómez Talleres. Albacete.

SANCHO CALATRAVA, J. A. (1989): Elecciones en la II Repüblica. Ciudad Real (19311936). Diputación Ciudad Real.

TUSELL, J. (1970): La II Reptiblica en Madrid: Elecciones y Partidos Polüticos. Madrid TUÑNON DE LARA, M. (1976) 3 edic. La II Repuiblica . Vol.II. p.1. Siglo XXI. Madrid. 